rite extensions were visualized with a microscope focused on the underside of the membrane (Figure 2B). Alternatively, neurites can be stained with a neuronal-specific, fluorescent-labeled antibody, such as $\beta$ IIItubulin (1:100; Chemicon International) (Figure 2A). For quantification of neurite extension, the dye was extracted from crystal violet-stained neurites. First, the cell bodies and neurites were separated from each other to quantify neurites only. Cell bodies were removed from the top chamber (on top of membrane) using a cotton swab (note that all cells and debris should be removed from the top, especially around edges of membrane where it attaches to plastic chamber). A 100 - to $200-\mu \mathrm{L}$ drop of extraction buffer $(25 \% 0.2 \mathrm{M}$ acetate buffer, $\mathrm{pH}$ $4.5,50 \%$ reagent alcohol) was placed on a flat piece of Parafilm ${ }^{\circledR}$. The underside of membrane, containing stained neurites, was placed on top of the drop of extraction buffer so that it covered the entire membrane surface. After a 5min incubation at room temperature, the 100 - to $200-\mu \mathrm{L}$ drop of extraction buffer (containing extracted crystal violet solution) was dispensed into a well of a 96-well plate and quantified at 562 $\mathrm{nm}$ on a spectrophotometer (Figure 2C).

BSA-coated $(0.2 \%)$ membranes were used as negative controls for neurite outgrowth of N1E-115 and PC12 cells. These controls were performed concurrently with assays done using ECMcoated membranes.

According to our results, N1E-115 and PC12 cells extended neurites on laminin- and collagen I-coated membranes, respectively, and could be visualized with either a fluorescently labeled neuronal antibody (Figure $2 \mathrm{~A}$ ) or crystal violet stain (Figure 2B). Crystal violet stain did not reveal any neurite outgrowth when cells were cultured on a BSA-coated membrane (Figure 2B, right panel). Figure $2 \mathrm{C}$ reveals quantification of neurite outgrowth from N1E115 cells, cultured on laminin and BSA-coated membranes, over a 24-h period. Neurite outgrowth at each time point was quantified at $562 \mathrm{~nm}$ following extraction of crystal violet stain from neurites.

In conclusion, the assay presented here is a simple and efficient method for the quantification of neurite outgrowth in a 24-well format. This system will also be applicable for the simultaneous screening of numerous biological and pharmacological agents, the direct evaluation of adhesion and guidance receptor functions responsible for neurite extension and repulsion, and the analysis of gene function in transfected cells.

\section{REFERENCES}

1.Qui, J., D. Cai, and M.T. Filbin. 2000. Glial inhibition of nerve regeneration in mature mammalian CNS. Glia 29:166-174.

2.Fournier, A.E. and S.M. Strittmatter. 2001 Repulsive factors and axon regeneration in the CNS. Curr. Opin. Neurobiol. 11:89-94.

3.Filbin, M.T. 2000. Axon regeneration: vaccinating against spinal cord injury. Curr. Biol. 10:R100-R103.

4.McKerracher, L. 2001. Spinal cord repair: strategies to promote axon regeneration. Neurobiol. Dis. 8:11-18.

5.Schmid, R.S., W.M. Pruitt, and P.F. Maness. 2000. A MAP kinase-signaling pathway mediates neurite outgrowth on L1 and requires Src-dependent endocytosis. J. Neurosci. 20:4177-4188.

6.Ronn, L.C., I. Ralets, B.P. Hartz, M. Bech, A. Berezin, V. Berezin, A. Moller, and E. Bock. 2000. A simple procedure for quantification of neurite outgrowth based on stereological principles. J. Neurosci. Methods
100:25-32

7.Boyden, S. 1963. Cellular recognition of foreign matter. Int. Rev. Exp. Pathol. 2:311-357.

8.Letourneau, P.C. 1975. Cell-to-substratum adhesion and guidance of axonal elongation. Dev. Biol. 44:92-101.

9.Gunderson, R.W. and J.N. Barrett. 1984. Neurite growth cone-substratum adherence increases in vitro. Brain Res. 314:21-26.

10.Lander, A.D., D.K. Fujii, and L.F. Reichardt. 1985. Purification of a factor that promotes neurite outgrowth: isolation of laminin and associated molecules. J. Cell Biol. 101:898-913.

11.Lander, A.D., D.K. Fujii, and L.F. Reichardt. 1985. Laminin is associated with the "neurite outgrowth-promoting factors" found in conditioned media. Proc. Natl. Acad. Sci. USA 82:2183-2187.

12.Turner, D.C., L.A. Flier, and S. Carbonetto. 1989. Identification of a cell-surface protein involved in PC12 cell-substratum adhesion and neurite outgrowth on laminin and collagen. J. Neurosci. 9:3287-3296.

Received 10 March 2003; accepted 29 April 2003.

Address correspondence to Richard Klemke, Innovative Cell Systems LLC, 28835 Single Oak Drive, Suite 110, Temecula, CA 92590, USA.e-mail:rklemke@innovativecell.com

\title{
Catechol dioxygenase is an insensitive reporter for transcription in Mycobacterium smegmatis
}

\author{
Shyam Unniraman ${ }^{1}$ and Valakunja Nagaraja ${ }^{1,2}$ \\ 'Indian Institute of Science and '2Jawaharlal Nehru Centre for Advanced Scientific \\ Research, Bangalore, India
}

BioTechniques 35:256-262 (August 2003)

The whole genome sequencing initiative has provided a wealth of information about mycobacteria $(1,2)$. However, inadequate genetic reagents continue to hinder rapid progress in the field. For instance, the analysis of gene expression in mycobacteria is acutely limited by the lack of sensitive and robust reporter systems. Over the years, there have been isolated reports of the use of a few reporter systems (3-7). However, because of the limited use of individual reporter systems, there is no comparison of the pros and cons of these systems specifically in mycobacteria, unlike in other model organisms such as Escherichia coli. Recently, the systems based on chloramphenicol acetyl transferase (CAT) (8-13) and catechol dioxygenase (CDO, xylE) (6,14-16) have become increasingly popular. The cat reporter gene has been used in many different systems and is well documented to be highly sensitive with an exceptionally large range of linearity. On the negative side, as with 
any antibiotic selection, the whole-cell assay is lethal and therefore requires the more cumbersome replica plating. Furthermore, the quantitative assays require the use of radioactivity and preparation of cell lysates. In contrast, the CDO system allows for color-based whole-cell assays and a simple quantitative assay that give comparable results in both whole cells and extracts. In addition, since the $x y l E$ gene is from Pseudomonas, which is highly GCrich, it might be better suited for mycobacteria than the CAT system. However, little is known about the sensitivity and linearity of the CDO reporter system. Therefore, here we present a comparison between the CAT and $\mathrm{CDO}$ systems as reporters for the analysis of different features of gene expression in mycobacteria.

The vectors pSD7 (5) and pTKmx (14), which contain promoterless cat and $x y l E$ genes, respectively, were used for all experiments. Both are E. coli-mycobacteria shuttle vectors containing kanamycin resistance gene as a selectable marker and the pAL5000 origin of replication. Furthermore, these two vectors are highly homologous in their construction, and both of them have transcription terminators on either side of the reporter gene, thereby insulating the reporter gene from other vector transcripts. These features make them ideal for comparison between the two re- porter genes. The construction of the pSD7-based plasmids pTUN1, 3 and 4 (11), pSUN7 (10), and pTER5 (12) have been described previously. Equivalent constructs were generated in the pTK$\mathrm{mx}$ context by cloning the same fragments into the BamHI site upstream of the $x y l E$ gene. The termination constructs were generated by PCR-amplifying putative transcription terminators from downstream of the Mycobacterium tuberculosis tuf and Rv1324 genes (12) and cloning them downstream of the promoter in pTER5 (and its pTKmx equivalent). All analyses were performed using the Mycobacterium smegmatis strain mc $^{2} 155$ as the mycobacterial host. The CAT and CDO assays were performed as described previously $(6,10)$. Briefly, for CAT activity, extracts were prepared from cells in mid-log phase $\left(\mathrm{A}_{595}=1\right)$ by sonication. Appropriate dilutions of the extracts were incubated with radioactive chloramphenicol and acetyl coenzyme A at $37^{\circ} \mathrm{C}$ for $30 \mathrm{~min}$. The reaction was stopped by extraction with ethyl acetate, and the products were resolved by thin layer chromatography. The CAT activity was normalized to total protein amount. The CDO activity was measured in whole cells (mid-log phase) by spectrophotometrically following the conversion of catechol to 2-hydroxymuconic semialdehyde at $375 \mathrm{~nm}$. The CDO activity was normalized to the $\mathrm{A}_{595}$ of the cul-

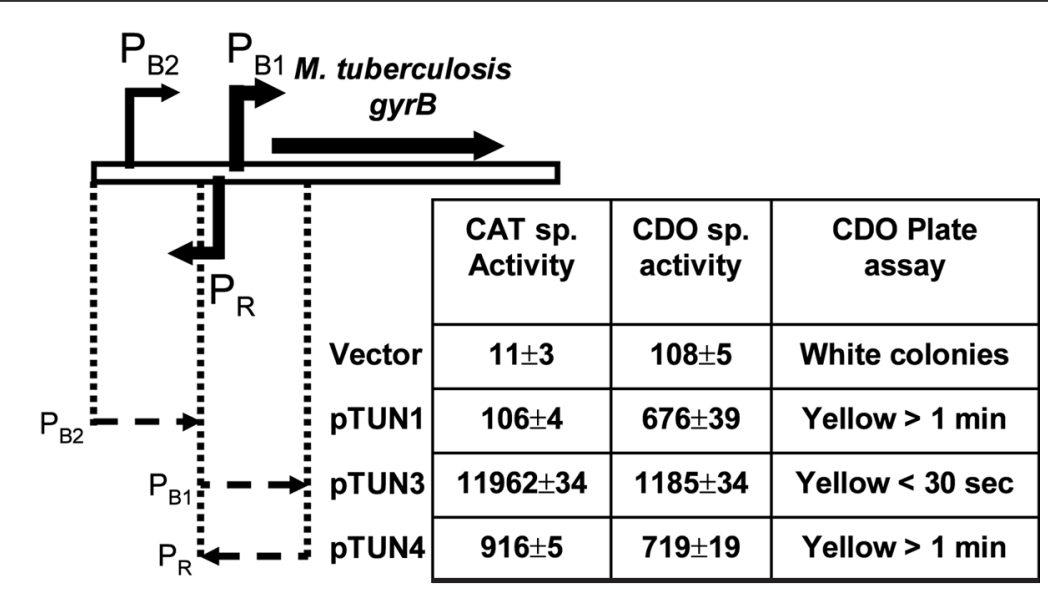

Figure 1. Assessing relative promoter strengths. The CAT- and CDO-based vectors and constructs harboring individual promoters were assessed for their respective reporter activity. The semiquantitative plate assay for CDO-based constructs is also shown. The location of the promoters upstream of $M$. tuberculosis gyrB and the fragments used to generate the clones is shown. The names of vectors in the context of the cat system are shown. Equivalent constructs in the $x y l E$ context were used to assess CDO activity. ture. For the CDO plate assay, catechol was sprayed on colonies, and the development of the yellow-colored product was followed visually.

We analyzed the sensitivity and specificity to these two reporter systems in three contexts: $(i)$ the difference in basal transcription from different promoters, (ii) induction of transcription, and (iii) transcription termination. To determine their sensitivity to intrinsic differences in promoter strength, we tested the promoters present upstream of the gyrB gene in $M$. tuberculosis that show a 100-fold range in relative strengths as determined by CAT activity (11) (Figure 1). It is noteworthy that this difference in promoter strength is reflected both in the level of CAT protein as assessed by immunoblotting and the chloramphenicol resistance in plate assays (data not shown). However, these promoters show a significantly reduced range with the $\mathrm{CDO}$ reporter system with the strongest promoter $\left(\mathrm{P}_{\mathrm{B} 1}\right)$ appearing to be less than 2-fold stronger than the weakest promoter $\left(\mathrm{P}_{\mathrm{B} 2}\right)$. Furthermore, because of the overall reduction in signal strength, the background activity seen with the promoterless vector is significantly higher $(9.1 \%$ of pTUN3) compared to the CAT system $(0.09 \%$ of pTUN3). Notably, in plate assays, all promoter-containing clones appeared positive, while colonies harboring the vector were negative, and there was some correlation in the rate of color development with the quantitative measure of CDO activity.

Next, we analyzed the transcriptional induction of the $M$. smegmatis gyrase promoter. The gyrase promoter in $M$. smegmatis is induced 6- to 7-fold when cells are treated with novobiocin (10) (Figure 2). Novobiocin is a potent inhibitor of DNA gyrase (17) and induces global relaxation of the genome (18). However, in the CDO reporter system, there was no detectable induction of transcription at any concentration of novobiocin (Figure 2).

Finally, we analyzed the ability of the CDO system to detect the functioning of transcriptional terminators. Previously, we have shown that both classical rho-independent terminators and atypical terminators function efficiently in mycobacteria (12). Towards this end, we cloned two different transcrip- 
tional terminators between the $M$. smegmatis gyrase promoter and the reporter gene. The premature termination of the transcript reduces read-through to the reporter gene, reflecting in decreased reporter gene activity. Surprisingly, terminators that reduced read- through about 5- to 10-fold, as measured by the CAT reporter system (12), failed to show any significant effect in the CDO context (Figure 3).

Thus, while the convenience of a color-based whole-cell assay and inexpensive nonradioactive substrate makes the

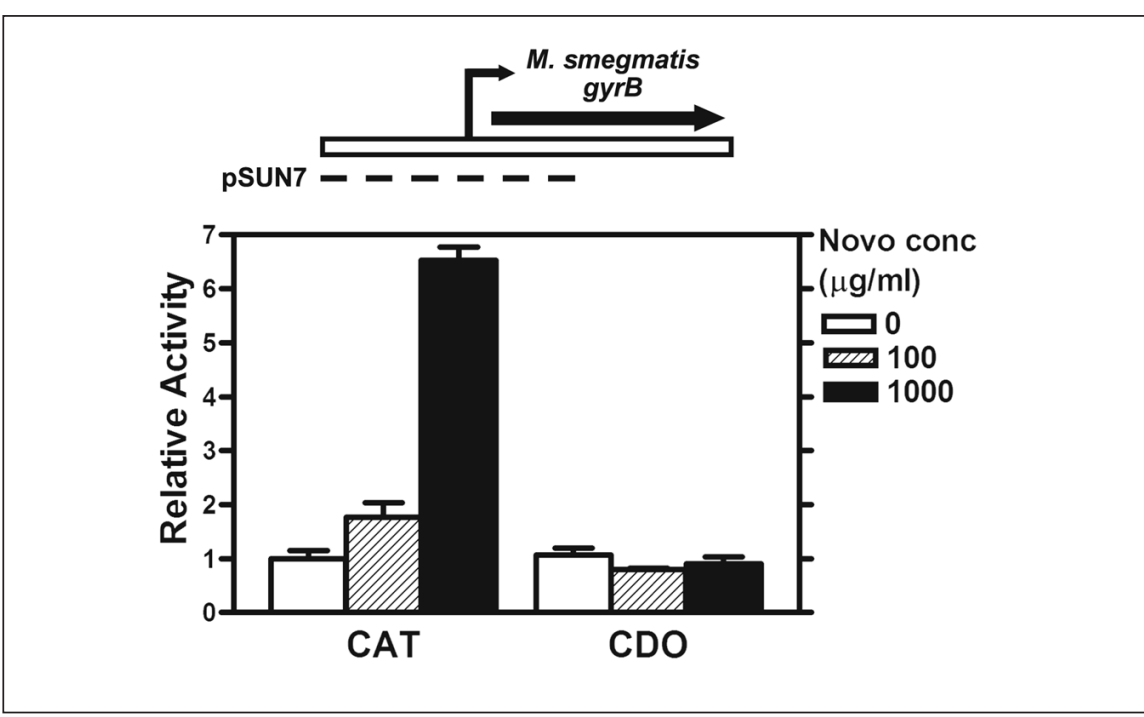

Figure 2. Assessing induction of transcription. Cells harboring the construct pSUN7 or its CDO equivalent were grown to mid-log phase and treated with different concentrations of novobiocin (Novo conc) as indicated. After $2 \mathrm{~h}$ treatment, cells were harvested, and reporter gene activity was measured as described in the text. The reporter activities are normalized with respect to the untreated cultures. The promoter fragment used to generate these constructs is shown above.

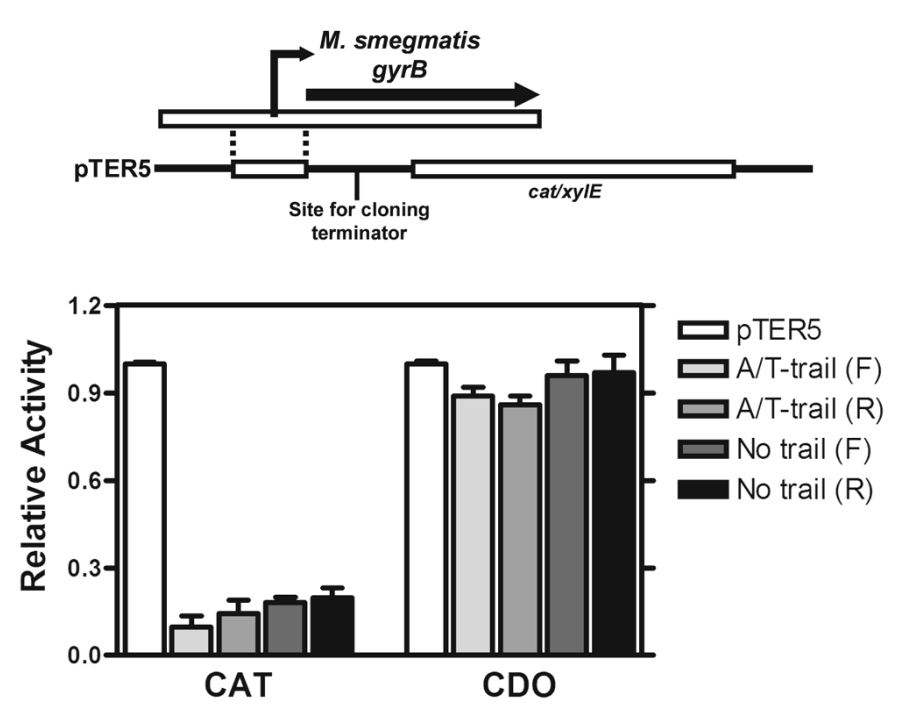

Figure 3. Assessing termination efficiency. The terminator selection vector pTER 5 and the constructs harboring different terminators in either orientation between the promoter and the cat gene have been described previously (12). Putative terminators were PCR-amplified downstream of M. tuberculosis tuf (A/T-trail) and Rv1324 (No trail) genes. Equivalent constructs were generated in the CDO context as well. Cells harboring these constructs were grown to mid-log phase and harvested, and the respective reporter activities were assessed as described in the text. The reporter activities are normalized with respect to the promoter alone constructs.
CDO assay attractive, it has severe shortcomings as a quantitative measure of transcript levels. The results discussed above suggest that, unlike CAT, the CDO-based system appears to be significantly limited in its range. As a result, a 100-fold difference in promoter strengths is reduced to about 2-fold, and smaller alterations in transcription are not evident. On the positive side, the $\mathrm{CDO}$ reporter system is still reliable in detecting promoters in the plate assay, since even the weakest promoters showed a significant activity above the vector control. The limited quantitative range of the system is also seen in earlier reports $(14,16)$ wherein most of the mutations had a marginal or a crippling effect on the reporter activity and very few mutations showed intermediate levels of promoter activity. The reason for this blunted range of the CDO reporter is not clear. The two vectors - pSD7 and pTK$\mathrm{mx}$-harboring the reporters are very similar, and the only significant difference between them is the reporter gene itself. Thus, the most likely cause of this damped range in the CDO system arises from some feature of the coding region or the sequence flanking it. Therefore, we feel that it probably arises from an abnormally slow or rapid degradation of the protein. Slow degradation would lead to an accumulation of significant amounts of protein even with a weak promoter. In contrast, rapid degradation would prevent accumulation of the protein even when a strong promoter is present. Needless to say, other possibilities (e.g., altered stability of the message) or more complicated models (e.g., induction of degradative processes as more protein or message is made) cannot be excluded based on our results. In conclusion, the study presented here shows the importance of direct comparison of reporter systems for mycobacteria and the need to identify robust, convenient alternatives to the cumbersome albeit reliable CAT system. This study also shows the necessity to determine the range of reliability of reporter systems before their use in quantitative experiments.

\section{ACKNOWLEDGMENTS}

We thank Anil K. Tyagi (University of Delhi, Delhi, India) for pSD7 and G. 
Churchward (Emory University, Atlanta, GA, USA) for pTKmx. The work was funded by grants from the Indian Council of Medical Research.

\section{REFERENCES}

1.Cole, S.T., R. Brosch, J. Parkhill, T. Garnier, C. Churcher, D. Harris, S.V. Gordon, K. Eiglmeier, et al. 1998. Deciphering the biology of Mycobacterium tuberculosis from the complete genome sequence. Nature 393:537-544.

2.Cole, S.T., K. Eiglmeier, J. Parkhill, K.D. James, N.R. Thomson, P.R. Wheeler, N. Honore, T. Garnier, et al. 2001. Massive gene decay in the leprosy bacillus. Nature 409:1007-1011.

3.Thomas, T.J., R.E. Andrews, Jr., and C.O. Thoen. 1992. Molecular cloning and characterization of Mycobacterium paratuberculosis promoters in Escherichia coli. Vet. Microbiol. 32:351-362.

4.Triccas, J.A., F.X. Berthet, V. Pelicic, and B. Gicquel. 1999. Use of fluorescence induction and sucrose counterselection to identify $M y$ cobacterium tuberculosis genes expressed within host cells. Microbiology 145(Pt 10):2923-2930.

5.Das Gupta, S.K., M.D. Bashyam, and A.K. Tyagi. 1993. Cloning and assessment of mycobacterial promoters by using a plasmid shuttle vector. J. Bacteriol. 175:5186-5192.

6.Curcic, R., S. Dhandayuthapani, and V. Deretic. 1994. Gene expression in mycobacteria: transcriptional fusions based on $x y l E$ and analysis of the promoter region of the response regulator mtrA from Mycobacterium tuberculosis. Mol. Microbiol. 13:1057-1064.

7.Alland, D., A.J. Steyn, T. Weisbrod, K. Aldrich, and W.R. Jacobs, Jr. 2000. Characterization of the Mycobacterium tuberculosis iniBAC promoter, a promoter that responds to cell wall biosynthesis inhibition. J. Bacteriol. 182:1802-1811.

8.Bashyam, M.D., D. Kaushal, S.K. Dasgupta, and A.K. Tyagi. 1996. A study of mycobacterial transcriptional apparatus: identification of novel features in promoter elements. J. Bacteriol. 178:4847-4853.

9.Bashyam, M.D. and A.K. Tyagi. 1998. Identification and analysis of "extended -10 " promoters from mycobacteria. J. Bacteriol. 180:2568-2573.

10.Unniraman, S. and V. Nagaraja. 1999. Regulation of DNA gyrase operon in Mycobacterium smegmatis: a distinct mechanism of relaxation stimulated transcription. Genes Cells 4:697-706

11.Unniraman, S., M. Chatterji, and V. Nagaraja. 2002. DNA gyrase genes in Mycobacterium tuberculosis: a single operon driven by multiple promoters. J. Bacteriol. 184:54495456.

12.Unniraman, S., R. Prakash, and V. Nagaraja. 2001. Alternate paradigm for intrinsic transcription termination in eubacteria. J. Biol. Chem. 276:41850-41855.

13.Vasanthakrishna, M., N.V. Kumar, and U. Varshney. 1997. Characterization of the ini- tiator tRNA gene locus and identification of a strong promoter from Mycobacterium tuberculosis. Microbiology 143(Pt 11):3591-3598.

14.Kenney, T.J. and G. Churchward. 1996. Genetic analysis of the Mycobacterium smegmatis rpsL promoter. J. Bacteriol. 178:35643571.

15.Stolt, P., Q. Zhang, and S. Ehlers. 1999. Identification of promoter elements in mycobacteria: mutational analysis of a highly symmetric dual promoter directing the expression of replication genes of the Mycobacterium plasmid pAL5000. Nucleic Acids Res. 27:396-402.

16.Dastur, A. and U. Varshney. 2001. Promoter analysis in mycobacteria using $x y I E$ reporter assays and its implication in high throughpu screening. Tuberculosis (Edinb.) 81:267-269.

17.Gellert, M., M.H. O'Dea, T. Itoh, and J.
Tomizawa 1976 Novobiocin and coumermycin inhibit DNA supercoiling catalyzed by DNA gyrase. Proc. Natl. Acad. Sci. USA 73:4474-4478.

18.Drlica, K. and M. Snyder. 1978. Superhelical Escherichia coli DNA: relaxation by coumermycin. J. Mol. Biol. 120:145-154.

Received 12 February 2003; accepted 1 May 2003.

Address correspondence to Dr. Valakunja Nagaraja, Department of Microbiology and Cell Biology, Indian Institute of Science, Bangalore-560012, India.e-mail:vraj@ mcbl.iisc.ernet.in

\title{
Quantification of DNA probes on nylon microarrays using $\mathrm{T} 4$ polynucleotide kinase labeling
}

\author{
M. El Atifi', I. Dupré2, B. Rostaing1, A.L. Benabid ${ }^{2,3}$, and F. Berger2,3 \\ ${ }^{1} \mathrm{CHRU}$, Equipe Transcriptome, INSERM U318, and '3niversité Joseph Fourier, \\ Grenoble, France
}

BioTechniques 35:262-266 (August 2003)

High-throughput gene expression profiling is a powerful tool for investigating transcriptional activity in many fields of biology and medicine (1-4). For microarray analysis, especially with radioactive detection, the relative quantification of the DNA probes spotted onto the membranes is required to perform the normalization of the expression level $(2,5)$ and to control the potential lack of probes on the filters.

Long oligonucleotide arrays have recently been described for glass array $(4,6,7)$ and nylon array methodologies (5). In a previous article (5), we described a 12-mer tag hybridization to quantify oligonucleotide probes onto filters. However, the tag synthesis at the extremity of all oligonucleotides generates a significant cost overrun and is unavailable for oligonucleotides that are included in commercial sets.

In this study, we describe and validate a new method for the quantifica- tion of DNA probes onto nylon filters using T4 polynucleotide kinase in the presence of radiolabeled $\left[\gamma_{-}{ }^{33} \mathrm{P}\right] \mathrm{ATP}$ to phosphorylate probes.

Oligonucleotides (from 40- to 70mers) without $5^{\prime}$ modification were spotted onto nylon filters and crosslinked using ultraviolet light exposure, as previously described (5). The quantification using sequence tag hybridization was performed according to our previous work (5).

The quantification using polynucleotide kinase reaction was performed as follows: the filters were prehybridized for $4 \mathrm{~h}$ at room temperature in $2 \mathrm{~mL} 200 \mathrm{mM}$ phosphate buffer $\left(\mathrm{Na}_{2} \mathrm{HPO}_{4} / \mathrm{NaH}_{2} \mathrm{PO}_{4}, \mathrm{pH} 7.4\right), 1 \mathrm{M}$ $\mathrm{NaCl}, 2 \%$ Tween ${ }^{\circledR} 20$, and $12 \%$ bovine serum albumin (BSA). The phosphorylation reaction was then performed for $12 \mathrm{~h}$ at room temperature with $30 \mu \mathrm{Ci}$ radiolabeled $\left[\gamma_{-}{ }^{33} \mathrm{P}\right] \mathrm{ATP}(3000 \mathrm{Ci} / \mathrm{mM}$; Amersham Biosciences, Little Chal- 